\title{
ARRANGEMENTS OF LINES AND MONODROMY OF ASSOCIATED MILNOR FIBERS
}

\author{
MARIO SALVETTI and MATTEO SERVENTI \\ Department of Mathematics, University of Pisa (Italy) \\ salvetti@dm.unipi.it, serventi@mail.dm.unipi.it
}

\begin{abstract}
Consider an arrangement $\mathcal{A}$ of homogeneous hyperplanes in $\mathbb{C}^{n}$, with complement $\mathcal{M}(\mathcal{A})$. The $(\mathrm{co})$ homology of $\mathcal{M}(\mathcal{A})$ with twisted coefficients is strictly related to the cohomology of the Milnor fibre associated to the natural fibration onto $\mathbb{C}^{*}$, endowed with the geometric monodromy. It is still an open problem to understand in general the cohomology of the Milnor fibre, even for dimension 1. In the first part we show that all questions about the first homology group are detected by a precise group, which is a quotient ot the commutator subgroup of $\pi_{1}(\mathcal{M}(\mathcal{A}))$ by the commutator of its length zero subgroup, which didn't appear in the literature before.

In the second part we state a conjecture of $a$-monodromicity for the first homology, which is of a different nature with respect to the known results. Let $\Gamma$ be the graph of double points of $\mathcal{A}$ : we conjecture that if $\Gamma$ is connected then the geometric monodromy acts trivially on the first homology of the Milnor fiber (so the first Betti number is combinatorially determined in this case). This conjecture depends only on the combinatorics of $\mathcal{A}$. We show the truth of the conjecture under some stronger hypotheses.
\end{abstract}

Keywords: arrangements of hyperplanes; monodromy, Milnor fiber

Mathematics Subject Classification 2000: 55N25; 57M05

\section{Introduction}

Let $\mathcal{A}:=\left\{\ell_{1}, \ldots, \ell_{n}\right\}$ be an arrangement of affine lines in $\mathbb{C}^{2}$, with complement $\mathcal{M}(\mathcal{A})$. By "coning" $\mathcal{A}$ one obtains a three-dimensional central arrangement $\widetilde{\mathcal{A}}$ in $\mathbb{C}^{3}$, with complement fibering over $\mathbb{C}^{*}$. The Milnor fiber $F$ of such fibration is a surface of degree $n+1$, endowed with a natural geometric monodromy automorphism of order $n+1$. It is well known that the trivial (co)homology of $F$ with coefficients in a commutative ring $A$, as a module over the monodromy action, is obtained by the (co)homology of $\mathcal{M}(\mathcal{A})$ with coefficients in $R:=A\left[t^{ \pm 1}\right]$, where here the structure of $R$ as a $\pi_{1}(\mathcal{M}(\mathcal{A})$ )-module is given by taking good generators (given by elementary loops around each hyperplane) into $t$-multiplication, and the monodromy action corresponds to $t$-multiplication. For reflection arrangements, relative to a Coxeter group $\mathbf{W}$, many computations were done, especially for the orbit space $\mathcal{M}_{\mathbf{W}}(\mathcal{A}):=\mathcal{M}(\mathcal{A}) / \mathbf{W}$, which has an associated Milnor fiber $F_{\mathbf{W}}:=F / \mathbf{W}:$ in this case we know a complete answer for $R=\mathbb{Q}\left[t^{ \pm 1}\right]$, for all groups of finite type 
(see $[20,12,13])$, and for some groups of affine type $([7,8,9])$ (based on the techniques developed in $[29,14])$. For $R=\mathbb{Z}\left[t^{ \pm 1}\right]$ a complete answer is known in case $A_{n}$ (see [6]). Some results are known for (non quotiented) reflection arrangements (see [30], [25]). (For related work on characteristic and resonance varieties see for ex. $[32,10,17,24,18,11])$.

It is still an open problem to understand the (co)-homology of the Milnor fiber $F$; e.g. it is not known if $H^{*}(F ; \mathbb{Z})$ is combinatorially determined (as it is wellknown to be the cohomology of the complement [26]). Even the first Betti number is not well-understood, so here we focus onto the first homology group of $F$. One sees that $b_{1}(F) \geq|\mathcal{A}|$, with equality holding iff the action of the monodromy is trivial (corollary 2.3). So, it is important to understand when there is some monodromy: we call an arrangement with trivial monodromy (in the first homology group) a-monodromic. Many of the known examples of arrangements with nontrivial monodromy are based on the theory of nets and multinets (see [21]): there are relatively few arrangements with non trivial monodromy and some conjecture claims very strict restrictions for line arrangements (see [34]).

We introduce a group which measures exactly the non-monodromicity of the arrangement. Let $\mathbf{G}=\pi_{1}(\mathcal{M}(\mathcal{A}))$ and let $\mathbf{K}$ be the kernel of the length map $\mathbf{G} \rightarrow \mathbb{Z}$; let us consider the group $\mathbf{G}^{\prime}:=\frac{[G, G]}{[K, K]}$. The main result of part 3 is that $H_{1}(F)$ is an extension of a free abelian group by the group $\mathbf{G}^{\prime}$ (thm. 1). So, any question about the first homology of $F$ is actually a question on $\mathbf{G}^{\prime}$. We can give an extimate for the first Betti number of $F$, as well as we deduce that if $\mathbf{G}$ is quasi-perfect then $\mathcal{A}$ is a-monodromic.

It seems that this group $\mathbf{G}^{\prime}$ was not considered before in this context.

In part 4 we consider a vanishing conjecture of a very different nature with respect to the known results, which is of combinatorial nature. Let $\Gamma$ be the graph with vertex set $\mathcal{A}$ and edge set which is given by taking an edge $\left(\ell_{i}, \ell_{j}\right)$ iff $\ell_{i} \cap \ell_{j}$ is a double point. Then our conjecture is as follows:

Conjecture: Assume that $\Gamma$ is connected; then $\mathcal{A}$ is a-monodromic.

This conjecture is supported by several computations which we made and all nontrivial monodromy examples which we know have disconnected graph $\Gamma$ (in part 4 we also mention how one can make a more technical and general conjecture). As a "canonical" example, the arrangement having equation $x y(x-1)(y-1)(x-y)=0$ has non-trivial monodromy and graph $\Gamma$ having three connected components.

We introduce some extra conditions for which we can prove the conjecture, giving examples in part 5 . Some more details will appear elsewhere. The main tool for our calculations is an algebraic complex computing the twisted cohomology of 
$\mathcal{M}(\mathcal{A})$ introduced in [22], as a simplification of a more general one introduced in [31] in connection with the minimal cell structure of the complement.

\section{Basic properties.}

Let $\mathcal{A}=\left\{H_{1}, \ldots, H_{n}\right\}$ be an arrangement of affine hyperplanes in $\mathbb{C}^{n}$, having coordinates $z_{1}, \ldots, z_{n}$. Let $\alpha_{i}=0$ be a linear defining equation for the hyperplane $H_{i}, i=1, \ldots, n$. The associated conified arrangement is the homogeneous arrangement $c \mathcal{A}=\left\{\widetilde{H_{0}}, \widetilde{H_{1}}, \ldots, \widetilde{H_{n}}\right\}$ in $\mathbb{C}^{n+1}$, with coordinates $z_{0}, \ldots, z_{n}$, where $\widetilde{H}_{0}$ is the coordinate hyperplane $z_{0}=0$ and $\widetilde{H}_{i}$ is the zero locus of the homogenization of $\alpha_{i}$ with respect to $z_{0}, 1 \leq i \leq n$.

Conversely, let $\widetilde{\mathcal{A}}=\left\{\widetilde{H}_{0}, \ldots, \widetilde{H}_{n}\right\}$ be a central arrangement in $\mathbb{C}^{n+1}$, with coordinates $z_{0}, \ldots, z_{n}$, such that $\widetilde{H}_{0}=\left\{z_{0}=0\right\}$ and for every $1 \leq i \leq n$, let $\widetilde{\alpha}_{i}\left(z_{0}, \ldots, z_{n}\right)=0$ be a defining equation for $\widetilde{H}_{i}$. Then the deconing of $\widetilde{\mathcal{A}}$ is the arrangement $d \widetilde{\mathcal{A}}=\left\{H_{1}, \ldots, H_{n}\right\}$ in $\mathbb{C}^{n}$ where $H_{i}$ is the zero locus of $\alpha_{i}$, with $\alpha_{i}\left(z_{1}, \ldots, z_{n}\right)=\widetilde{\alpha_{i}}\left(1, z_{1}, \ldots, z_{n}\right), 1 \leq i \leq n$ (see [26]).

We are interested to the topology of the complements of the arrangements, which we denote by $\mathcal{M}(A), \mathcal{M}(\widetilde{\mathcal{A}})$, etc. One easily checks the relation:

$$
\mathcal{M}(c \mathcal{A})=\mathcal{M}(\mathcal{A}) \times \mathbb{C}^{*} \quad\left(\text { iff } \mathcal{M}(\widetilde{\mathcal{A}})=\mathcal{M}(d \widetilde{\mathcal{A}}) \times \mathbb{C}^{*}\right) .
$$

Let $Q: \mathbb{C}^{n+1} \rightarrow \mathbb{C}$ be a homogeneous polynomial (of degree $n+1$ ) which defines the arrangement $\widetilde{\mathcal{A}}$ (the product of the linear forms $\widetilde{\alpha_{i}}$ 's). Then $Q$ gives a fibration

$$
Q_{\mid \mathcal{M}(\tilde{\mathcal{A}})}: \mathcal{M}(\tilde{\mathcal{A}}) \rightarrow \mathbb{C}^{*}
$$

with Milnor fibre

$$
\mathbf{F}=Q^{-1}(1)
$$

and geometric monodromy

$$
\pi_{1}\left(\mathbb{C}^{*}, 1\right) \rightarrow \operatorname{Aut}(F)
$$

induced by the map $x \rightarrow e^{\frac{2 \pi i}{n+1}} \cdot x$.

The fundamental group $\pi_{1}(\mathcal{M}(\widetilde{\mathcal{A}}))$ is generated by elementary loops $\beta_{i}, i=$ $0, \ldots, n$, going around the hyperplanes, well defined up to conjugacy; in the decomposition $\pi_{1}(\mathcal{M}(\widetilde{\mathcal{A}})) \simeq \pi_{1}(\mathcal{M}(d \mathcal{A})) \times \mathbb{Z}$ the generator of $\mathbb{Z}=\pi_{1}\left(\mathbb{C}^{*}\right)$ corresponds to a loop going around all the hyperplanes (the generators can be ordered so that such a loop is represented by the product $\left.\beta_{0} \ldots \beta_{n}\right)$.

Recall also that $H_{1}(\mathcal{M}(\widetilde{\mathcal{A}}) ; \mathbb{Z}) \cong \mathbb{Z}^{n+1}$, generated by the classes of the $\beta_{i}$ 's. The following representation is therefore well-defined.

Let $A$ be any unitary commutative ring and let

$$
R:=A\left[t, t^{-1}\right]
$$


be the ring of Laurent polynomials with coefficients in $A$. Consider the abelian representation

$$
\pi_{1}(\mathcal{M}(\widetilde{\mathcal{A}})) \rightarrow H_{1}(\mathcal{M}(\widetilde{\mathcal{A}}) ; \mathbb{Z}) \rightarrow \operatorname{Aut}(R): \beta_{j} \rightarrow t .
$$

taking a generator $\beta_{j}$ into $t$-multiplication. We denote by $R_{t}$ the ring $R$ endowed with this $\pi_{1}(\mathcal{M}(\widetilde{\mathcal{A}}))$-module structure. Then it is well-known:

Proposition 2.1. One has an R-module isomorphism

$$
H_{*}\left(\mathcal{M}(\widetilde{\mathcal{A}}), R_{t}\right) \cong H_{*}(F, A)
$$

where $t$-multiplication on the left corresponds to the monodromy action on the right.

We are interested in particular in the case $A=\mathbb{Z}$ or $A=\mathbb{Q}$. For $R=\mathbb{Q}\left[t, t^{-1}\right]$, which is a PID, one has

$$
H_{*}\left(\mathcal{M}(\widetilde{\mathcal{A}}), R_{t}\right) \cong H_{*}(F, \mathbb{Q}) .
$$

Since the monodromy operator has clearly order dividing $n+1$, one finds that $H_{*}\left(\mathcal{M}(\widetilde{\mathcal{A}}) ; R_{t}\right)$ decomposes into cyclic modules either isomorphic to $R$ or to $\frac{R}{\left(\varphi_{d}\right)}$, where $\varphi_{d}$ is the $d$ th-cyclotomic polynomial, for some $d \mid n+1$. It is an open problem to find a (possibly combinatorial) formula for the Betti numbers of $F$.

In this paper we are interested in the first homology group of the Milnor fibre.

From the spectral sequence associated to the bundle (2.1) we easily deduce

\section{Lemma 2.2.}

$$
n+1=\operatorname{dim}\left(H_{1}(\mathcal{M}(\widetilde{\mathcal{A}}) ; \mathbb{Q})\right)=1+\operatorname{dim} \frac{H_{1}(F ; \mathbb{Q})}{(\mu-1)}
$$

where on the right one has the coinvariants with respect to the monodromy action.

It follows

\section{Corollary 2.3.}

$$
b_{1}(F) \geq n
$$

and

$$
b_{1}(F)=n \quad \Leftrightarrow \quad \mu=i d .
$$


Definition 2.4. An arrangement $\widetilde{\mathcal{A}}$ with trivial monodromy (in the first homology group of $F$ with rational coefficients) will be briefly called a-monodromic.

One can extend definition 2.4 to arrangements which are a-monodromic over $A$, meaning that the monodromy over $H_{1}(F ; A)$ is trivial.

Remark 2.5. By (2.3) the arrangement $\widetilde{\mathcal{A}}$ is a-monodromic iff

$$
\left.H_{1}(F ; \mathbb{Q}) \cong \mathbb{Q}^{n} \text { (equivalently: } H_{1}\left(\mathcal{M}(\widetilde{\mathcal{A}}) ; R_{t}\right) \cong\left(\frac{R}{(t-1)}\right)^{n}\right) .
$$

We can introduce a similar concept for affine arrangements.

Definition 2.6. Say that the affine arrangement $\mathcal{A}$ is a-monodromic if

$$
H_{1}\left(\mathcal{M}(\mathcal{A}) ; R_{t}\right) \cong\left(\frac{R}{(t-1)}\right)^{n-1} .
$$

Let $\mathcal{A}=d \widetilde{\mathcal{A}}$ be the affine part. Notice that in the above decomposition $\mathcal{M}(\widetilde{\mathcal{A}})=$ $\mathcal{M}(d \widetilde{\mathcal{A}}) \times \mathbb{C}^{*}$ the generator of the fundamental group of the $\mathbb{C}^{*}$ factor correponds inside $\mathcal{M}(\widetilde{\mathcal{A}})$ to a loop turning around all the $n+1$ hyperplanes. Then one has an algebraic complex computing the twisted homology which factorizes as

$$
C_{*}\left(\mathcal{M}(\widetilde{\mathcal{A}}), R_{t}\right)=C_{*}\left(\mathcal{M}(d \widetilde{\mathcal{A}}), R_{t}\right) \otimes_{R} C_{*}\left(\mathbb{C}^{*}, R_{t}\right)
$$

where the action of $t$ on the right factor of the second member is multiplication by $t^{n+1}$. Therefore one easily obtains (with $A=\mathbb{Z}$ or $\mathbb{Q}$ )

$$
H_{1}\left(\mathcal{M}(\widetilde{\mathcal{A}}) ; R_{t}\right) \cong H_{1}\left(\mathcal{M}(\mathcal{A}) ; R_{t}\right) \otimes \frac{R}{\left(t^{n+1}-1\right)} \oplus \frac{R}{(t-1)} .
$$

As an easy example in dimension 1 , when $\widetilde{\mathcal{A}}$ is the central arrangement of three lines in $\mathbb{C}^{2}$ (with fundamental group $\pi_{1}\left(\mathcal{M}(\widetilde{\mathcal{A}})\right.$ ) the pure braid group $P_{3}$ ) then $d \widetilde{\mathcal{A}}$ is the affine two-points arrangement in $\mathbb{C}^{1}$. One easily sees that $H_{1}\left(\mathcal{M}(\mathcal{A}), R_{t}\right)=R$; moreover, $H_{1}\left(\mathcal{M}(\widetilde{\mathcal{A}}), R_{t}\right)=\frac{R}{(t-1)} \oplus \frac{R}{\left(t^{3}-1\right)}$ (see [22]) which agrees with formula (2.2).

It follows that if $\mathcal{A}$ has trivial monodromy (over $A=\mathbb{Z}$ or $\mathbb{Q}$ ) then $\widetilde{\mathcal{A}}$ does. The converse is not true in general (see fig 8 ).

\section{Intrinsic characterizations}

We give here a more intrinsic picture.

Recall that the fundamental group

$$
\mathbf{G}=\pi_{1}(\mathcal{M}(\widetilde{\mathcal{A}})) \quad\left(=\pi_{1}(\mathcal{M}(\mathcal{A})) \times \mathbb{Z}\right)
$$

is generated by elementary loops $\beta_{0}, \ldots, \beta_{n}$ around the hyperplanes.

Let

$$
\mathbf{F}=\mathbf{F}_{\mathbf{n}+\mathbf{1}}\left[\beta_{0}, \ldots, \beta_{n}\right]
$$


be the free group with basis the $\beta_{i}$ 's and let $\mathbf{N}$ be the normal subgroup generated by the relations. It is well-known that $\mathbf{G}$ is a commutator-relator group, i.e. $\mathbf{N} \subset[\mathbf{F}, \mathbf{F}]$ (see for ex. [28]).

One has a presentation

$$
1 \longrightarrow \mathbf{N} \longrightarrow \mathbf{F} \stackrel{\pi}{\longrightarrow} \mathbf{G} \longrightarrow 1
$$

The length map $\varphi: \mathbf{F} \rightarrow<t>\cong \mathbb{Z}$ is defined as

$$
\varphi(w)=t^{|w|} .
$$

Here if $w=\beta_{i_{1}}^{\epsilon_{1}} \ldots \beta_{i_{k}}^{\epsilon_{k}}$ is a reduced expression for $w \in \mathbf{F}$, we set $|w|=\sum_{1 \leq j \leq k} \epsilon_{i_{j}} \in$ $\mathbb{Z}$. Of course, $\varphi$ factors through the abelianization

$$
\varphi: \mathbf{F} \stackrel{a b}{\longrightarrow} \frac{\mathbf{F}}{[\mathbf{F}, \mathbf{F}]} \cong \mathbb{Z}^{n+1} \stackrel{\lambda}{\longrightarrow} \mathbb{Z}
$$

where $\lambda\left(a_{1}, \ldots, a_{n+1}\right)=\sum_{i} a_{i}$. Since all relations in $\mathbf{G}$ have zero length, $\varphi$ factors by $\pi$ through a map

$$
\psi: \mathbf{G} \stackrel{a b}{\longrightarrow} \frac{\mathbf{G}}{[\mathbf{G}, \mathbf{G}]} \cong \mathbb{Z}^{n+1} \stackrel{\mu}{\longrightarrow} \mathbb{Z}
$$

Let

$$
\mathbf{H}:=\operatorname{ker} \varphi ; \mathbf{K}=k e r \psi
$$

so we have

$$
1 \longrightarrow \mathbf{K} \longrightarrow \mathbf{G} \stackrel{\psi}{\longrightarrow} \mathbb{Z} \longrightarrow 1
$$

Lemma 3.1. One has

$$
\operatorname{ker}(\lambda)=\frac{\mathbf{H}}{[\mathbf{F}, \mathbf{F}]}, \quad \operatorname{ker}(\mu)=\frac{\mathbf{K}}{[\mathbf{G}, \mathbf{G}]}
$$

SO

$$
\frac{\mathbf{H}}{[\mathbf{F}, \mathbf{F}]} \cong \frac{\mathbf{K}}{[\mathbf{G}, \mathbf{G}]} \cong \mathbb{Z}^{n}
$$

Proof. First part derives from $[\mathbf{F}, \mathbf{F}] \subset \operatorname{ker}(\varphi)$ and $[\mathbf{G}, \mathbf{G}] \subset \mathbf{K}$.

Second part follows from $\frac{\mathbf{F}}{[\mathbf{F}, \mathbf{F}]} \cong \frac{\mathbf{G}}{[\mathbf{G}, \mathbf{G}]} \cong \mathbb{Z}^{n+1}$.

Recall (see proposition 2.1) the $\mathbb{Z}\left[t^{ \pm 1}\right]$-module isomorphism:

$$
H_{1}\left(\mathbf{G} ; \mathbb{Z}\left[t^{ \pm 1}\right]\right) \cong H_{1}(F ; \mathbb{Z})
$$


where $F$ is the Milnor fibre. By Shapiro Lemma (see [5]) one has

$$
H_{1}(F ; \mathbb{Z}) \cong H_{1}(\mathbf{K} ; \mathbb{Z})=\frac{\mathbf{K}}{[\mathbf{K}, \mathbf{K}]}
$$

Therefore we have:

Theorem 1. There is a (split) exact sequence

$$
1 \longrightarrow \frac{[\mathbf{G}, \mathbf{G}]}{[\mathbf{K}, \mathbf{K}]} \longrightarrow \frac{\mathbf{K}}{[\mathbf{K}, \mathbf{K}]} \longrightarrow \frac{\mathbf{K}}{[\mathbf{G}, \mathbf{G}]} \cong \mathbb{Z}^{n} \longrightarrow 1
$$

From the definition it follows

Lemma 3.2. The arrangement $\widetilde{\mathcal{A}}$ is a-monodromic over $\mathbb{Z}$ iff

$$
H_{1}(F ; \mathbb{Z}) \cong \mathbb{Z}^{n}
$$

Therefore

Theorem 2. The arrangement $\widetilde{\mathcal{A}}$ is a-monodromic over $\mathbb{Z}$ iff

$$
\frac{[\mathbf{G}, \mathbf{G}]}{[\mathbf{K}, \mathbf{K}]}=0
$$

Proof. It follows from sequence (3.4) and from the property that a surjective endomorphism of a finitely generated free abelian group is an isomorphism.

Since $\mathbf{K} \supset[\mathbf{G}, \mathbf{G}]$ it follows immediately

Corollary 3.3. Assume

$$
\mathbf{G}^{(1)}=[\mathbf{G}, \mathbf{G}]=\mathbf{G}^{(2)}=[[\mathbf{G}, \mathbf{G}],[\mathbf{G}, \mathbf{G}]]
$$

Then the arrangement $\widetilde{\mathcal{A}}$ is a-monodromic over $\mathbb{Z}$. 
A group $\mathbf{G}$ such that $\mathbf{G}^{(1)}=\mathbf{G}^{(2)}$ is called quasi-perfect.

Another consequence, easily deduced from theorem 2 is:

Corollary 3.4. Let $\mathbf{G}$ have a central element of length 1 . Then the arrangement $\widetilde{\mathcal{A}}$ is a-monodromic over $\mathbb{Z}$.

Proof. Let $\gamma \in \mathbf{G}$ be a central element of length 1. From sequence (3.1) the group splits as a direct product

$$
\mathbf{G} \cong \mathbf{K} \times \mathbb{Z}
$$

where $\mathbb{Z}=\langle\gamma\rangle$. Therefore clearly $[\mathbf{G}, \mathbf{G}]=[\mathbf{K}, \mathbf{K}]$.

An example of corollary (3.4) is when one of the generators $\beta_{j}$ commutes with all the others; this happens if one of the hyperplanes is transversal to all the others. So we re-find in this way a well-known fact.

Consider again the exact sequence (3.4). Recall that the arrangement $\widetilde{\mathcal{A}}$ is amonodromic $($ over $\mathbb{Q})$ iff $H_{1}(F ; \mathbb{Q}) \cong \mathbb{Q}^{n}$. By tensoring sequence $(3.4)$ by $\mathbb{Q}$ we obtain

\section{Theorem 3.}

(1) The arrangement $\widetilde{\mathcal{A}}$ is a-monodromic (over $\mathbb{Q}$ ) iff

$$
\frac{[\mathbf{G}, \mathbf{G}]}{[\mathbf{K}, \mathbf{K}]} \otimes \mathbb{Q}=0 .
$$

(2) $\operatorname{tors}\left(H_{1}(F)\right)=\operatorname{tors}\left(\frac{[\mathbf{G}, \mathbf{G}]}{[\mathbf{K}, \mathbf{K}]}\right)$.

(3) $b_{1}(F)=n+r k\left(\frac{[\mathbf{G}, \mathbf{G}]}{[\mathbf{K}, \mathbf{K}]}\right)$

(There are no examples where $F$ has torsion in the $H_{1}$. There are only complicated examples with torsion in higher homology, see [15]).

By using that $[\mathbf{G}, \mathbf{G}] \subset \mathbf{K}$ we immediately get the estimate:

Corollary 3.5. One has

$$
n \leq b_{1}(F) \leq n+r k\left(\frac{\mathbf{G}^{(1)}}{\mathbf{G}^{(2)}}\right)
$$

Remark 3.6. The preceding results apply as well to any commutator-relator group $\mathbf{G}$. Here we say that the group $\mathbf{G}$ is a-monodromic iff the action of $\mathbb{Z}$ onto $\mathbf{K}$ (see sequence (3.1)) induces the identity on the homology group $H_{1}(\mathbf{K})$. 
Now we consider again the affine arrangement $\mathcal{A}$. Denoting by $\mathbf{G}^{\prime}:=\pi_{1}(\mathcal{M}(\mathcal{A}))$, we have

$$
\mathbf{G} \cong \mathbf{G}^{\prime} \times \mathbb{Z}
$$

where the factor $\mathbb{Z}$ is generated by a loop around all the hyperplanes in $\widetilde{\mathcal{A}}$. The a-monodromicity of $\mathcal{A}$ (over $\mathbb{Z}$ ) is equivalent to

$$
H_{1}\left(\mathcal{M}(\mathcal{A}) ; R_{t}\right) \cong\left(\frac{R}{(t-1)}\right)^{n-1}
$$

$\left(R=\mathbb{Z}\left[q^{ \pm 1}\right]\right)$. By considering a sequence as in (3.1)

$$
1 \longrightarrow \mathbf{K}^{\prime} \longrightarrow \mathbf{G}^{\prime} \stackrel{\psi}{\longrightarrow} \mathbb{Z} \longrightarrow 1
$$

we can repeat the above arguments: in particular condition (3.6) is equivalent to

$$
H_{1}\left(\mathbf{K}^{\prime} ; \mathbb{Z}\right)=\frac{\mathbf{K}^{\prime}}{\left[\mathbf{K}^{\prime}, \mathbf{K}^{\prime}\right]}=\mathbb{Z}^{n-1}
$$

and we get an exact sequence like in (3.4) for $\mathbf{K}^{\prime}$ and $\mathbf{G}^{\prime}$. So we obtain

Theorem 4. The arrangement $\mathcal{A}$ is a-monodromic over $\mathbb{Z}$ (resp. over $\mathbb{Q}$ ) iff

$$
\frac{\left[\mathbf{G}^{\prime}, \mathbf{G}^{\prime}\right]}{\left[\mathbf{K}^{\prime}, \mathbf{K}^{\prime}\right]}=0\left(\text { resp. } \frac{\left[\mathbf{G}^{\prime}, \mathbf{G}^{\prime}\right]}{\left[\mathbf{K}^{\prime}, \mathbf{K}^{\prime}\right]} \otimes \mathbb{Q}=0\right) .
$$

By considering a presentation for $\mathbf{G}^{\prime}$

$$
1 \longrightarrow \mathbf{N}^{\prime} \longrightarrow \mathbf{F}^{\prime} \stackrel{\pi}{\longrightarrow} \mathbf{G}^{\prime} \longrightarrow 1
$$

where $\mathbf{F}^{\prime}$ is the group freely generated by $\beta_{1}, \ldots, \beta_{n}$, we obtain

Theorem 5. There is an isomorphism:

$$
\frac{\left[\mathbf{G}^{\prime}, \mathbf{G}^{\prime}\right]}{\left[\mathbf{K}^{\prime}, \mathbf{K}^{\prime}\right]} \cong \frac{\left[\mathbf{F}^{\prime}, \mathbf{F}^{\prime}\right]}{\mathbf{N}^{\prime}[\operatorname{ker} \varphi, \text { ker } \varphi]}
$$

so $\mathcal{A}$ is a-monodromic over $\mathbb{Z}$ (resp. over $\mathbb{Q}$ ) iff

$$
\frac{\left[\mathbf{F}^{\prime}, \mathbf{F}^{\prime}\right]}{\mathbf{N}^{\prime}[\operatorname{ker} \varphi, k e r \varphi]}=0\left(\text { resp. } \frac{\left[\mathbf{F}^{\prime}, \mathbf{F}^{\prime}\right]}{\mathbf{N}^{\prime}[\operatorname{ker} \varphi, k e r \varphi]} \otimes \mathbb{Q}=0\right)
$$

Proof. From

$$
\mathbf{N}^{\prime} \subset\left[\mathbf{F}^{\prime}, \mathbf{F}^{\prime}\right] \subset \operatorname{ker} \varphi
$$

it follows:

$$
\frac{\left[\mathbf{G}^{\prime}, \mathbf{G}^{\prime}\right]}{\left[\mathbf{K}^{\prime}, \mathbf{K}^{\prime}\right]} \cong \frac{\pi^{-1}\left[\mathbf{G}^{\prime}, \mathbf{G}^{\prime}\right]}{\pi^{-1}\left[\mathbf{K}^{\prime}, \mathbf{K}^{\prime}\right]} \cong \frac{\left[\mathbf{F}^{\prime}, \mathbf{F}^{\prime}\right]}{\mathbf{N}^{\prime}[\operatorname{ker} \varphi, \operatorname{ker} \varphi]}
$$




\section{Strategies for the conjecture}

First we mention how our conjecture can be generalized in a more technical setting. We refer to [18],[32] for the main definitions and constructions related to the characteristic varieties and resonance varieties of an arrangement.

It is easy to see that $\Gamma$ connected implies there are no essential components in the first resonance variety of $\widetilde{\mathcal{A}}$. Then $\widetilde{\mathcal{A}}$ is a-monodromic unless there is a translated component in the characteristic variety of $\widetilde{\mathcal{A}}$. Therefore a natural stronger conjecture is:

- assume that $\Gamma$ is connected: then there are no translated components in the (first) characteristic variety of the arrangement.

Our computations support also this stronger conjecture.

From now on we assume the arrangement is 2 -dimensional and affine. The main tool to make explicit computations is here an algebraic complex which was obtained in [22], as a 2-dimensional refinement of that in [31], where the authors used the explicit construction of a minimal cell complex which models the complement. Such complexes are defined in case of real defined arrangements: so we restrict ourself to this condition.

The complex depends on a fixed and generic system of "polar coordinates". In the present situation, this just means to take an oriented affine real line $\ell$ which is transverse to the arrangement. We also assume that $\ell$ is "far away" from $\mathcal{A}$, meaning that it does not intersect the closure of the bounded facets of the arrangement. This is clearly possible because the union of bounded chambers is a compact set (the arrangement is finite). The choice of $\ell$ induces a labelling on the lines $\left\{\ell_{1}, \ldots, \ell_{n}\right\}$ in $\mathcal{A}$, where the indices of the lines agree with the ordering of the intersection points with $\ell$, induced by the orientation of $\ell$.

Let us choose a basepoint $O \in \ell$, coming before all the intersection points of $\ell$ with $\mathcal{A}$ (with respect to the just introduced ordering). We recall the construction in $[22]$ in the case of the abelian local system defined before.

Let $\operatorname{Sing}(\mathcal{A})$ be the set of singular points of the arrangement. For any point $P \in \operatorname{Sing}(\mathcal{A})$, let $S(P):=\{\ell \in \mathcal{A}: P \in \ell\} ;$ so $m(P)=|S(P)|$ is the multiplicity of $P$.

Let $i_{P}, i^{P}$ be the minimum and maximum index of the lines in $S(P)$ (so $i_{P}<$ $\left.i^{P}\right)$. We denote by $C(P)$ the subset of lines in $\mathcal{A}$ whose indices belong to the closed interval $\left[i_{P}, i^{P}\right]$. We also denote by

$$
U(P):=\{\ell \in \mathcal{A}: \ell \text { does not separate } P \text { from the basepoint } O\}
$$

Let $\left(\mathcal{C}_{*}, \partial_{*}\right)$ be the 2 -dimensional algebraic complex of free $R$-modules having one 0 -dimensional basis element $e^{0}, \quad n 1$-dimensional basis elements $e_{j}^{1}, j=1, \ldots, n,\left(e_{j}^{1}\right.$ corresponding to the line $\left.\ell_{j}\right)$ and $\nu_{2}=\sum_{P \in \operatorname{Sing}(\mathcal{A})} m(P)-1$ 2 -dimensional basis elements: to the singular point $P$ of multiplicity $m(P)$ we as- 
sociate generators $e_{P, h}^{2}, h=1, \ldots, m(P)-1$. The lines through $P$ will be indicized as $\ell_{j_{P, 1}}, \ldots, \ell_{j_{P, m(P)}}$ (with growing indices).

From [22], we obtain after specialization:

Theorem 6. The local system homology $H_{*}(\mathcal{M}(\mathcal{A}) ; R)$ is computed by the complex $\left(\mathcal{C}_{*}, \partial_{*}\right)$ above, where

$$
\partial_{1}\left(e_{j}^{1}\right)=(t-1) e^{0}
$$

and

$$
\begin{aligned}
& \partial_{2}\left(e_{P, h}^{2}\right)=\sum_{\substack{\ell_{j} \in S(P) \\
j \geq j_{P}, h+1}} t^{\#\left\{\ell_{i} \in U(P): i<j\right\}+\#\left\{\ell_{i} \in S(P): j_{P, h+1} \leq i<j\right\}}\left(1-t^{\#\left\{\ell_{i} \in S(P): i \leq j_{P, h}\right\}}\right) e_{j}^{1}+ \\
& +\sum_{\substack{\ell_{j} \in S(P) \\
j \leq j_{P, h}}}^{\#\left\{\ell_{i} \in U(P): i<j\right\}+\#\left\{\ell_{i} \in S(P): i<j\right\}}\left(t^{\#\left\{\ell_{i} \in S(P): j_{P, h+1} \leq i\right\}}-1\right) e_{j}^{1}+ \\
& +\sum_{\substack{\ell_{j} \in C(P) \cap U(P)\\
}} t^{\#\left\{\ell_{i} \in U(P): i<j\right\}+\#\left\{\ell_{i} \in S(P): i \geq j_{P, h+1}, i<j\right\}}\left(1-t^{\#\left\{\ell_{i} \in S(P): i \leq j_{P, h}, i<j\right\}}\right) . \\
& \cdot\left(1-t^{\#\left\{\ell_{i} \in S(P): i \geq j_{P, h+1}, i \geq j\right\}}\right) e_{j}^{1}
\end{aligned}
$$

When $P$ is a double point, then $h$ takes only the value 1 , and $j_{P, 1}, j_{P, 2}$ are the indices of the two lines passing through $P$. So formula (4.1) becomes

$$
\begin{aligned}
& \partial_{2}\left(e_{P, 1}^{2}\right)=t^{\#\left\{\ell_{i} \in U(P): i<j_{P, 2}\right\}}(1-t) e_{j_{P, 2}}^{1}+t^{\#\left\{\ell_{i} \in U(P): i<j_{P, 1}\right\}}(t-1) e_{j_{P, 1}}^{1}+ \\
& +\sum_{\ell_{j} \in C(P) \cap U(P)} t^{\#\left\{\ell_{i} \in U(P): i<j\right\}}(t-1)^{2} e_{j}^{1}
\end{aligned}
$$

Since $\partial_{2}$ is divisible by $1-t$ we can rewrite (4.2) as

$$
\partial_{2}\left(e_{P, 1}^{2}\right)=(1-t) \tilde{\partial}_{2}\left(e_{P, 1}^{2}\right)
$$

where

$$
\begin{aligned}
& \tilde{\partial}_{2}\left(e_{P, 1}^{2}\right)=t^{\#\left\{\ell_{i} \in U(P): i<j_{P, 2}\right\}} e_{j_{P, 2}}^{1}-t^{\#\left\{\ell_{i} \in U(P): i<j_{P, 1}\right\}} e_{j_{P, 1}}^{1}+ \\
& +\sum_{\ell_{j} \in C(P) \cap U(P)} t^{\#\left\{\ell_{i} \in U(P): i<j\right\}}(1-t) e_{j}^{1}
\end{aligned}
$$


We now give a proof of conjecture 1 under stronger hypotheses.

Definition 4.1. We say that a subset $\Sigma$ of the set of $\operatorname{singular}$ points $\operatorname{Sing}(\mathcal{A})$ of the arrangement $\mathcal{A}$ is conjugate-free (with respect to a given admissible coordinate system) if $\forall P \in \Sigma$ the set $U(P) \cap C(P)$ is empty.

An arrangement $\mathcal{A}$ will be called conjugate-free if $\Gamma$ is connected and contains a spanning tree $T$ such that the set of points in $\operatorname{Sing}(\mathcal{A})$ that correspond to the edges $E T$ of $T$ is conjugate-free (see fig 5 ).

Let $\Sigma$ be conjugate-free: it follows from formula (4.1) that the boundary of all generators $e_{P, h}^{2}, P \in \Sigma$, can have non-vanishing components only along the lines which contain $P$.

Theorem 7. Assume that $\mathcal{A}$ is conjugate-free. Then $\mathcal{A}$ is a-monodromic.

Proof. The submatrix of $\tilde{\partial}_{2}$ which corresponds to the double points $E T$ is the incidence matrix of the tree $T$. Such matrix is the boundary matrix of the complex which computes the $\mathbb{Z}$-homology of $T$ : it is a unimodular rank- $(n-1)$ integral matrix (see for example [3]). From this the result follows straightforward.

We will also consider different situations: the proofs in these cases will appear elsewhere.

Definition 4.2. For $T$ an arbitrary spanning tree of $\Gamma$, we say that the induced labelling on $V T=V \Gamma$ is very good (with respect to the given coordinate system) if the sequence $n, \ldots, 1$ is a collapsing ordering on $T$. In other words, the graph obtained from $T$ by removing all vertices with label $\geq i$ and all edges having both vertices with label $\geq i$, is a tree, for all $i=n, \ldots, 1$.

We say that the spanning tree $T$ is very good if there exists an admissible coordinate system such that the induced labelling on $V T$ is very good (see fig 1).

\section{Remark 4.3.}

(1) A labelling over a spanning tree $T$ gives a collapsing ordering iff for each vertex $v$, the number of adjacent vertices with lower label is $\leq 1$. In this case, only the vertex labelled with 1 has no lower labelled adjacent vertices (by the connectness of $T$ ).

(2) Given a collapsing ordering over $T$, for each vertex $v$ with label $i_{v}>1$, let $\ell(v)$ be the edge which connects $v$ with the unique adjacent vertex with lower label; by giving to $\ell(v)$ the label $i_{v}+\frac{1}{2}$, we obtain a discrete Morse function on the graph $T$ (see [19]) with unique critical cell given by the vertex with label 1 . The set of all pairs $(v, \ell(v))$ is the acyclic matching which is associated to this Morse function. 
Let us indicate by $\Gamma_{0}$ the linear tree with $n$ vertices: we think as $\Gamma_{0}$ as a $C W$ decomposition of the real segment $[1, n]$, with vertices $\{j\}, j=1, \ldots, n$, and edges the segments $[j, j+1], j=1, \ldots, n-1$.

Definition 4.4. We say that a labelling induced by some coordinate system on the tree $T$ is good if there exists a permutation $i_{1}, \ldots, i_{n}$ of $1, \ldots, n$ which gives a collapsing sequence both for $T$ and for $\Gamma_{0}$. In other words, at each step we always remove either the maximum labelled vertex or the minimum, and this is a collapsing sequence for $T$.

We say that $T$ is good if there exists an admissible coordinate system such that the induced labelling on $V T$ is good (see fig 3 ).

Notice that a very good labelling is a good labelling where at each step one removes the maximum vertex.

Consider some arrangement $\mathcal{A}$ with graph $\Gamma$ and labels on the vertices which are induced by some coordinate system. Notice that changes of coordinates act on the labels by giving all possible cyclic permutations, which are generated by the transformation $i \rightarrow i+1 \bmod n$. So, given a labelled tree $T$, checking if $T$ is very good (resp. good) consists in verifying if some cyclic permutation of the labels is very good (resp. good). This property depends not only on the "shape" of the tree, but also on how the lines are disposed in $\mathbb{R}^{2}$ (the associated oriented matroid). In fact, one can easily find arrangements where some "linear" tree is very good, and others where some linear tree is not good.

Definition 4.5. We say that an arrangement $\mathcal{A}$ is very good (resp. good) if $\Gamma$ is connected and has a very good (resp. good) spanning tree.

It is not clear if this property is combinatorial, i.e. if it depends only on the lattice. Of course, $\mathcal{A}$ very good implies $\mathcal{A}$ good.

By simplifying the 2-boundary of the above complex one obtains:

Theorem 8. Let $\mathcal{A}$ be a good arrangement. Then $\mathcal{A}$ is a-monodromic.

We can have a mixed situation between definitions 4.5 and 4.1 (see fig 6).

Theorem 9. Assume that $\Gamma$ is connected and contains a spanning tree $T$ which reduces, after a sequence of moves where we remove either the maximum or the minimum labelled vertex, to a subtree $T^{\prime}$ which is conjugate-free. Then $\mathcal{A}$ is amonodromic.

Some examples are given in the following section. 
14 Mario Salvetti and Matteo Serventi

\section{Examples}

Recall that the vertices of the graph $\Gamma$ correspond to the lines of the arrangement. In the examples below the specified spanning trees are subgraphs of $\Gamma$. The edges of such trees correspond to certain double intersection points in the arrangement, indicated by heavy dots in the pictures.

In the first example (fig.1 and 2) we give an arrangement together with a very good tree and its sequence of contractions.

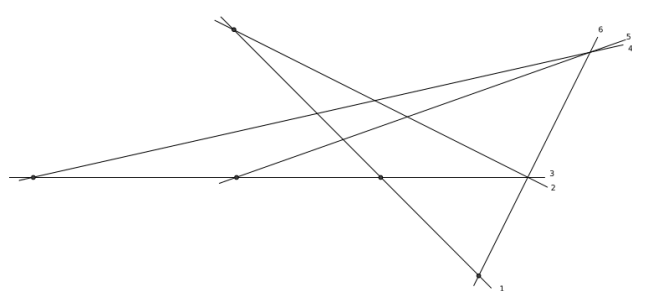

Figure 1. A very good tree

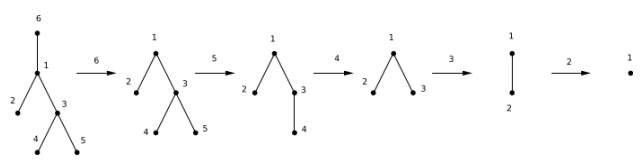

Figure 2. contractions 
Next (fig. 3,4$)$ we give a good tree with a sequence of contractions

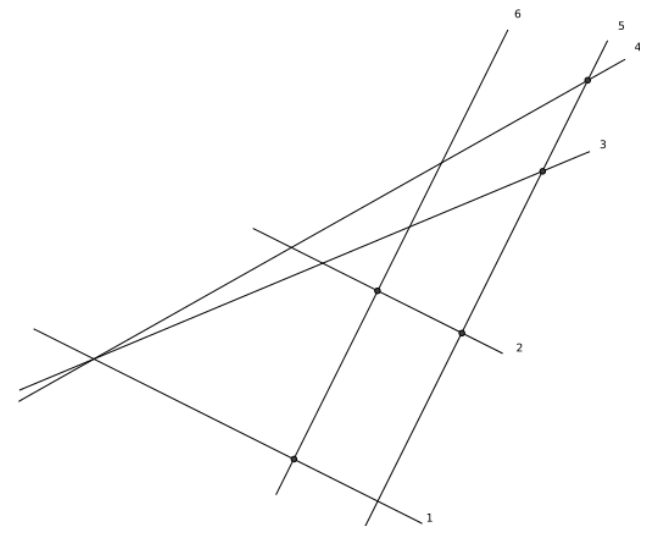

Figure 3. A very good tree

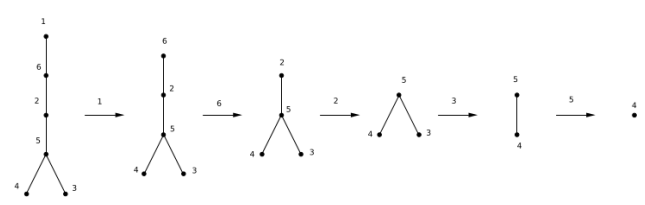

Figure 4. contractions 


\section{Mario Salvetti and Matteo Serventi}

and (fig.5) a conjugate free tree which is not good.
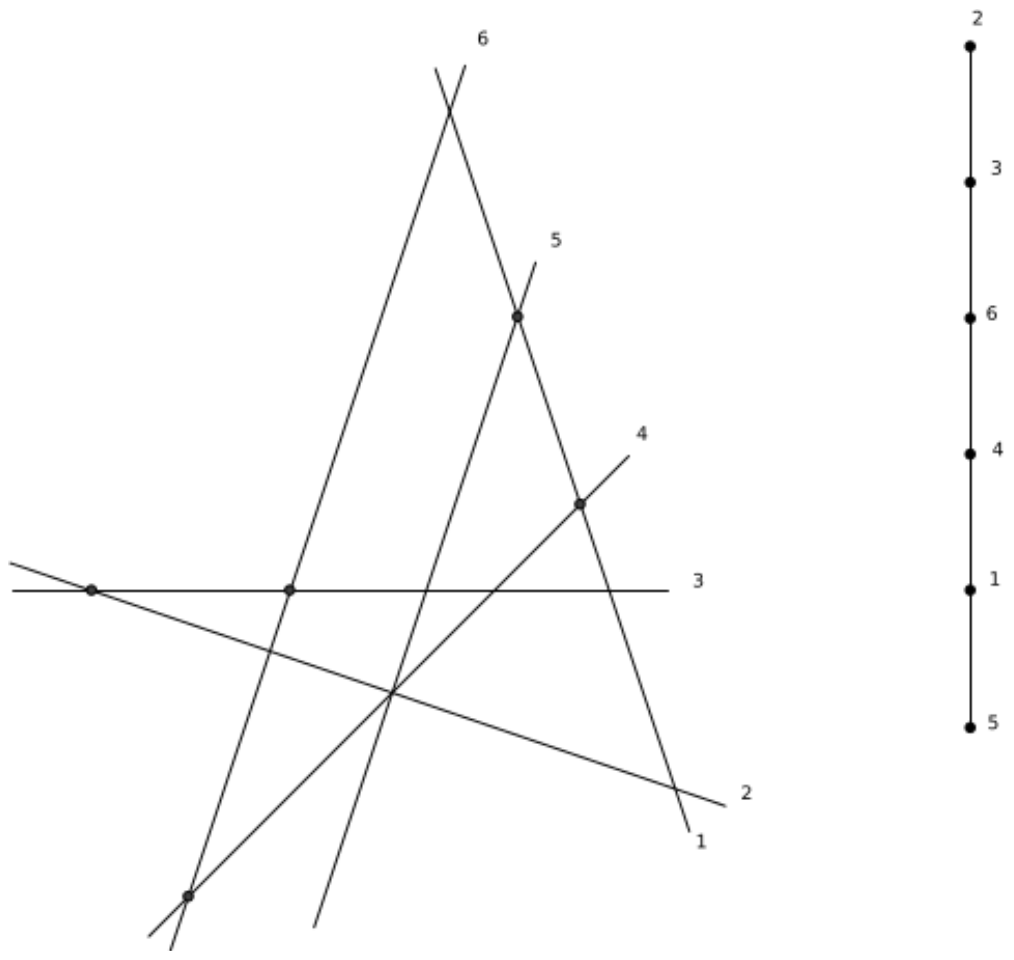

Figure 5. A conjugate free tree 
In fig. 6,7 , we have a tree which is not good but reduces to a conjugate free tree after two admissible moves.

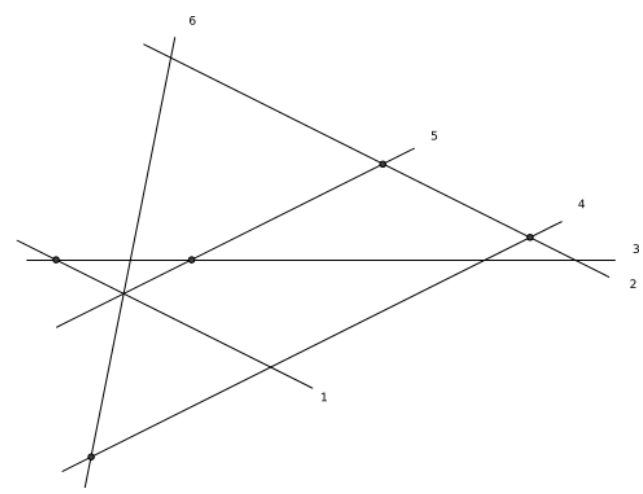

Figure 6. A very good tree

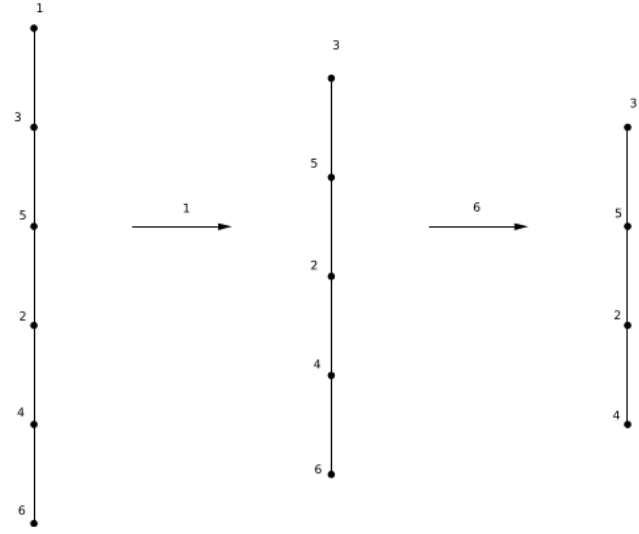

Figure 7. contractions 
In fig. 8 the affine complete triangle has $\varphi_{3}$-monodromy but its conified arrangement is $a$-monodromic. The computations of $H_{1}\left(\mathcal{M}(\mathcal{A}), R_{t}\right)$ are done by using the algebraic complex of theorem (6) as well as some computer calculations.
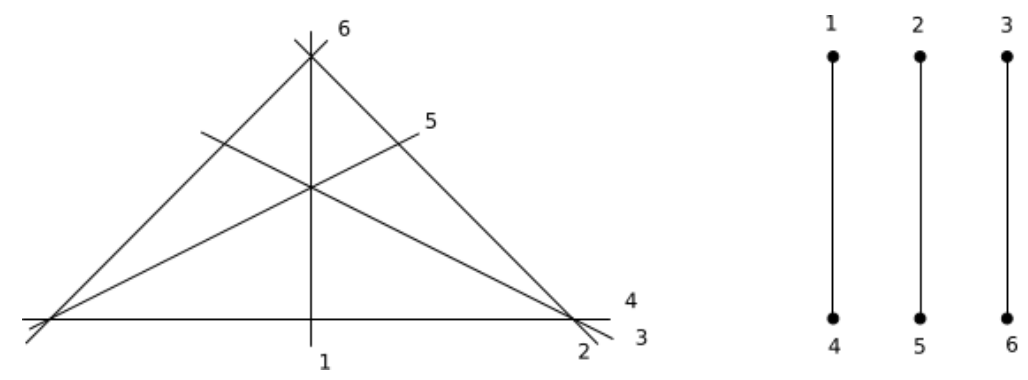

Figure 8 .

$$
\begin{gathered}
H_{1}\left(\mathcal{M}(\mathcal{A}), R_{t}\right) \simeq\left(\frac{\mathbb{Q}\left[t^{ \pm 1}\right]}{(t-1)}\right)^{4} \oplus \frac{\mathbb{Q}\left[t^{ \pm 1}\right]}{\left(t^{3}-1\right)} \\
H_{1}\left(\mathcal{M}(c \mathcal{A}), R_{t}\right) \simeq\left(\frac{\mathbb{Q}\left[t^{ \pm 1}\right]}{(t-1)}\right)^{6}
\end{gathered}
$$

\section{Acknowledgments}

Partially supported by INdAM and by: Università di Pisa under the "PRA Progetti di Ricerca di Ateneo" (Institutional Research Grants) - Project no. PRA_2016_67 "Geometria, Algebra e Combinatoria di Spazi di Moduli e Configurazioni". 


\section{Bibliography}

[1] D. Arapura, Geometry of cohomology support loci for local systems I, J. Alg. Geom. 6 (1997), 563-597.

[2] P. Bailet, On the monodromy of the Milnor fiber of hyperplane arrangements, arXiv:1401.6042v2[math.AG]

[3] N. Biggs, Algebraic graph theory, Cambridge University Press (1974).

[4] J. Birman, Braids, Links and Mapping Class Groups, Princeton Univ. Press (1975)

[5] K. Brown, Cohomology of Groups, GTM (1985)

[6] F. Callegaro, The homology of the Milnor fibre for classical braid groups Algeb. Geom. Topol. 6 (2006) 1903-1923

[7] F. Callegaro, D. Moroni and M. Salvetti, "Cohomology of Artin groups of type $\tilde{A}_{n}, \tilde{B}_{n}$ and applications", Geom \& Top. Mon. 13 (2008), 85-104.

[8] F. Callegaro, D. Moroni and M. Salvetti, Cohomology of affine Artin groups and applications, Trans. Amer. Mat. Soc. 360 (2008) 4169-4188.

[9] F. Callegaro, D. Moroni and M. Salvetti, The $K(\pi, 1)$ problem for the affine Artin group of type $\tilde{B}_{n}$ and its cohomology Jour. Eur. Math. Soc. 12 (2010) 1-22

[10] D. Cohen, P. Orlik, Arrangements and local systems, Math. Res. Lett. 7 (2000), no.2-2, 299-316.

[11] D. Cohen, A. Suciu, Characteristic varieties of arrangements, Math. Proc. Cambridge Philos. Soc. 127 (1999), 33-53.

[12] C. De Concini, C. Procesi, and M. Salvetti, Arithmetic properties of the cohomology of braid groups, Topology 40 (2001), no. 4, 739-751.

[13] C. De Concini, C. Procesi, M. Salvetti, and F. Stumbo, Arithmetic properties of the cohomology of Artin groups, Ann. Scuola Norm. Sup. Pisa Cl. Sci. (4) 28 (1999), no. $4,695-717$.

[14] C. De Concini and M. Salvetti, Cohomology of Artin groups, Math. Res. Lett. 3 (1996), no. 2, 293-297.

[15] G. Denham, A. Suciu, Multinets, parallel connections, and Milnor fibrations of Arrangements, arXiv:1209.3414[math.AG]

[16] A. Dimca, S. Papadima, Hypersurface complements, Milnor fibers and higher homotopy groups of arrangements, Ann. of Math. (2) 158 (2003), no.2, 473-507.

[17] A. Dimca, S. Papadima and A. Suciu Topology and geometry of cohomology jump loci, Duke Math. J. 148 (2009), 405-457.

[18] M.J. Falk, Arrangements and cohomology, Ann. Combin. 1 (2) (1997) 135-157.

[19] R. Forman, Morse Theory for Cell Complexes, Adv. in Math. 134 (1998), no.1, 90-145.

[20] E.V. Frenkel, Cohomology of the commutator subgroup of the braid group, Func. Anal. Appl. 22 (1988), no.13, 248-250.

[21] M. J. Falk and S. Yuzvinsky Multinets, resonance varieties and pencils of plane curves, Comp. Math. 143 (2007), 10069-1088.

[22] G. Gaiffi and M. Salvetti The Morse complex of a line arrangement, Jour. of Algebra 321 (2009), 316-337.

[23] G. Gaiffi, F. Mori and M. Salvetti Minimal CW-complexes for Complement to Line Arrangements via Discrete Morse Theory, Topology of Alg Var. and Sing., AMS, COntemporary Math., 538 (2011), 293-308.

[24] A. Libgober, S. Yuzvinsky, Cohomology of the Orlik-Solomon algebras and local systems, Compositio Math. 21 (2000), 337-361.

[25] A. Mùacinic, S. Papadima, On the monodromy action on Milnor fibers of graphic ar- rangements, Topology Appl. 156 (2009), no. 4, 76-774. 
[26] P. Orlik, M. Terao, Arrangements of hyperplanes, Springer-Verlag 300 (1992).

[27] R. Randell, Morse theory, Milnor fibers and minimality of a complex hyperplane arrangement, Proc. Amer. Math. Soc. 130 (2002), no. 9, 2737-2743

[28] M. Salvetti, Topology of the complement of real hyperplanes in $\mathbb{C}^{n}$, Inv. Math., 88 (1987), no.3, 603-618.

[29] M. Salvetti, The homotopy type of Artin groups, Math. Res. Lett. 1 (1994), 567-577.

[30] S. Settepanella, Cohomology of Pure Braid Groups of exceptional cases, Topol. and Appl. 156 (2009), 1008-1012.

[31] M. Salvetti, S. Settepanella, Combinatorial Morse theory and minimality of hyperplane arrangements, Geometry \& Topology 11 (2007) 1733-1766.

[32] A. Suciu, Translated tori in the characteristic varieties of complex hyperplane arrangements, Topol. Appl. 118 (2002) 209-223

[33] A. Suciu, arXiv:1502.02279

[34] M. Yoshinaga, Resonant bands and local system cohomology groups for real line arrangements Vietnam J. Math. 42 (2014), no.3, 377-392 